Quim. Nova, Vol. 35, No. 1, 124-131, 2012

\title{
SÍNTESE E CARACTERIZAÇÃO DE BEADS ACRÍLICOS PREPARADOS POR POLIMERIZAÇÃO EM SUSPENSÃO VISANDO APLICAÇÃO COMO EXCIPIENTE FARMACÊUTICO PARA COMPRESSÃO DIRETA
}

\author{
Janaina Cecília Oliveira Villanova* \\ Faculdade de Farmácia, Universidade de Mogi das Cruzes, Campus Villa-Lobos, Av. Imperatriz Leopoldina, 550, \\ 05305-000 São Paulo - SP, Brasil \\ Tadeu Henrique Lima \\ Escola de Engenharia, Departamento de Engenharia Metalúrgica e de Minas, Universidade Federal de Minas Gerais, Belo \\ Horizonte - MG, Brasil \\ Patrícia Santiago Patrício \\ Departamento de Química, Centro Federal de Educação Tecnológica de Minas Gerais, Belo Horizonte - MG, Brasil \\ Fabiano Vargas Pereira \\ Departamento de Química, Universidade Federal de Minas Gerais, Belo Horizonte - MG, Brasil \\ Eliane Ayres \\ Escola de Design, Departamento de Materiais, Tecnologia e Processos, Universidade Estadual de Minas Gerais, Belo Horizonte \\ - MG, Brasil
}

Recebido em 1/3/11; aceito em 27/6/11; publicado na web em 5/8/11

\begin{abstract}
SYNTHESIS AND CHARACTERIZATION OF ACRYLIC BEADS OBTAINED BY SUSPENSION POLYMERIZATION PROCESS FOR USE AS PHARMACEUTICAL EXCIPIENT FOR DIRECT COMPRESSION. One of the difficulties reported for the suspension polymerization is control the size and granulometry of beads. The purpose of this work was to evaluate the use of cellulose nanowhiskers and mesoporous silica as stabilizers to reduce the size and the particle distribution. To monitor polymerization process was used FTIR-ATR spectroscopy. The morphology was analyzed by scanning electron microscopy. The particle size distribution was characterised using a CILAS granulometer. Thermal stability was studied by thermogravimetric analysis. The results indicated that cellulose nanowhiskers may provide stabilization and increase the thermal stability of the beads in contrast to mesoporous silica.
\end{abstract}

Keywords: suspension polymerization; stabilizers agents; acrylic pharmaceutical excipients.

\section{INTRODUÇÃO}

Excipiente é qualquer substância (exceto o fármaco ou o prófármaco) que tem sua segurança avaliada e, a partir de então, pode ser incluído no medicamento, com intenções variadas, tais como, auxiliar na preparação; fornecer estabilidade física, química e microbiológica ao produto; garantir a aceitabilidade do paciente; e, melhorar ou promover qualquer outro atributo relacionado à segurança e efetividade do medicamento. ${ }^{1}$

Em decorrência dos avanços no delineamento de novos sistemas de liberação de fármacos (SLFs), inclusive de comprimidos, faz-se necessário que sejam desenvolvidos novos excipientes, idealizados para exercerem funções específicas nas formulações, tais como, melhorar a biodisponibilidade do fármaco e otimizar as características de manufaturabilidade dos comprimidos bem como de outras formas farmacêuticas. ${ }^{2-7}$ Portanto, é cada vez maior na literatura o número de artigos que relatam pesquisas voltadas para o desenvolvimento de novos excipientes farmacêuticos. ${ }^{8-17}$

A maioria dos comprimidos de liberação prolongada disponíveis comercialmente contém hidroxipropilmetilcelulose (HPMC) como agente formador de matriz. Porém, o mecanismo de gelificação da HPMC, que controla a liberação do fármaco, nem sempre é ideal para modular a cedência de fármacos muito solúveis. Muitas vezes, grandes quantidades de HPMC são necessárias. Outro problema é que a HPMC não possui características de fluxo e compressão adequados à compressão direta e, a preparação de grânulos, pela via úmida, pode gerar partículas rígidas. ${ }^{18,19}$

\footnotetext{
*e-mail: janainavillanova@umc.br
}

Polímeros acrílicos encontram-se disponíveis para a produção de comprimidos a partir de diversas vias. A técnica de revestimento é um método trabalhoso, que envolve grande número de etapas, componentes e, em alguns casos, o uso de solventes orgânicos..$^{20-22}$ Excipientes acrílicos disponíveis para a preparação de comprimidos matriciais empregando a granulação úmida caracterizam-se por serem dispersões aquosas ou orgânicas, com baixo teor em sólidos (cerca de 40\%), o que leva à necessidade de utilizar grande quantidade do polímero, inviabilizando a formulação. Aqueles disponíveis na forma sólida nem sempre possuem características adequadas à compressão direta. ${ }^{23-25}$

Contudo, para que a compressão direta seja a via de produção de escolha para os comprimidos, faz-se necessário que sejam empregados excipientes apropriados, chamados de diretamente compressíveis. Estes, por sua vez, precisam apresentar atributos em conformidade com as especificações de qualidade, segurança e funcionalidade. Portanto, devem possuir propriedades físicas adequadas, tais como, fluidez e compressibilidade. Algumas das análises preconizadas para a avaliação destas propriedades são denominadas micromeríticas, e referem-se à ciência que investiga características como forma das partículas, diâmetro, distribuição de tamanho, densidade, área superficial, carga superficial, entre outras. ${ }^{2,3}$

Neste sentido, o objetivo principal do presente trabalho foi desenvolver um excipiente acrílico, na forma sólida, a ser utilizado na preparação de comprimidos matriciais inertes por compressão direta. Os monômeros selecionados para o preparo dos copolímeros foram o acrilato de etila (EA), o metacrilato de metila (MMA) e o metacrilato de butila (BMA). A seleção foi baseada no fato de que os monômeros acrílicos são moléculas altamente reativas e versáteis, que podem 
interagir entre si e com outras moléculas, originando homopolímeros e copolímeros com propriedades físicas e químicas adequadas à dada necessidade. ${ }^{19,26,27}$ Ainda, a seleção foi fundamentada no conhecimento da composição das dispersões poliméricas de uso farmacêutico, disponíveis comercialmente, uma vez que as monografias das mesmas encontram-se inscritas nas Farmacopeias. ${ }^{28,29}$

A polimerização em suspensão foi a técnica selecionada para a preparação do excipiente por ser um método que fornece polímeros no estado sólido, na forma de pérolas (esferas ou beads). O processo é caracterizado pela presença de um ou mais monômeros relativamente insolúveis na fase aquosa e de um iniciador solúvel na fase orgânica. O início é térmico e radicalar, ocorrendo na gota de monômero. A quantidade usual do iniciador varia de 0,1 a $0,5 \%$ $\mathrm{p} / \mathrm{p}$ em relação à massa dos monômeros, que devem ser dispersos na fase aquosa pela combinação de agitação forte e uso de agentes suspensores e estabilizantes. A estabilidade pode ser alcançada por diferentes mecanismos, entre eles, por estabilização estérea e/ ou eletrostática. ${ }^{30-32}$

O processo de polimerização em suspensão apresenta inúmeras vantagens quando comparado a outras técnicas. $\mathrm{O}$ controle eficiente da temperatura durante a reação, devido à eficiente remoção do calor, em decorrência da baixa viscosidade do meio, é a principal. Além disso, o produto final tem elevada pureza e a separação é simples, podendo ser feita por sedimentação e filtração. Outra vantagem é que a polimerização ocorre com altas taxas de conversão dos monômeros. Finalmente, a polimerização em suspensão é mais rápida, envolve menos etapas e possui custo mais baixo se comparada a outros métodos. ${ }^{30-34}$

A principal dificuldade relatada na literatura para a técnica em suspensão é o controle do tamanho das partículas e da sua distribuição granulométrica, o que pode ser conseguido pelo ajuste de parâmetros como frequência de agitação, controle da temperatura de reação, tipo de reator e composição do sistema. ${ }^{31-40}$ Nestes casos, a alternativa mais estudada é a introdução de estabilizantes na formulação. Inúmeros são os agentes empregados no processo, cabendo destacar o uso do poli(álcool vinílico), da poli(pirrolidona vinílica), do poli(ácido acrílico), de argilas, gomas naturais e derivados da celulose, tais como a HPMC e a celulose microcristalina (CMC). A estabilização é atribuída à proteção coloidal. ${ }^{37,41-43}$ Alguns agentes atuam por meio de um fenômeno denominado Pickering. Estes estabilizantes são sólidos insolúveis, com tamanho submicrométrico, que possuem molhabilidade intermediária entre a fase aquosa e a orgânica. As partículas sofrem adsorção nas gotas da fase dispersa, formando uma barreira capaz de promover estabilização estérea ou diminuindo a tensão superficial, por redução no ângulo de contato. Dessa forma, evitam a coalescência e mantêm as gotículas formadas. ${ }^{44-50}$ Partículas como a sílica, a laponita, a montmorilonita, os carbonatos e óxidos nanoparticulados vêm sendo utilizadas como estabilizantes por Pickering. ${ }^{44,51-55}$

Assim, além do desenvolvimento de um novo excipiente, outro objetivo deste trabalho foi investigar a estabilidade de sistemas de polimerização em suspensão empregando diferentes tipos de estabilizantes, nomeadamente, os nanocristais de celulose e a sílica mesoporosa. Apesar de diversos trabalhos que abordam tal tema, não foram encontrados relatos na literatura técnica e científica acerca do emprego dos agentes selecionados. Celulose nanowhiskers (CNW), também denominada de nanocristais de celulose (CNC), são nanoestruturas em forma de bastonetes, altamente cristalinas, obtidas a partir da hidrólise ácida controlada da celulose, processo que destrói as regiões amorfas das fibras, deixando os segmentos cristalinos intactos e levando à formação de cristais de elevada pureza. As dimensões dos nanocristais dependem, principalmente, da fonte de celulose e das condições de hidrólise. Para a maioria dos nanocristais obtidos de fibras vegetais o comprimento é de cerca de 150-300 nm e diâmetro de 4-10 nm. ${ }^{56,57}$ As nanofibras de celulose possuem inúmeras vantagens em relação a outros materiais nanoestruturados, como facilidade no processo de obtenção, baixo custo da matéria-prima, características diversificadas em função do substrato natural de origem e obtenção a partir de fontes renováveis. ${ }^{56,57}$

O outro agente estabilizante pesquisado foi a MCM-41, uma das peneiras moleculares mais estudadas. As peneiras moleculares constituem uma família de materiais porosos que possuem elevada capacidade de troca iônica e adsorção e permitem a difusão seletiva de moléculas em seu interior. A MCM-41 é sintetizada a partir de silicatos em fase aquosa na presença de surfactantes, gerando estruturas altamente organizadas e com elevada área superficial. Segundo a IUPAC, os materiais mesoporosos se caracterizam por apresentarem diâmetros de poros entre 2 e $54 \mathrm{~nm}$. A MCM-41 possui arranjo hexagonal de mesoporos e sistemas de poros unidimensionais. ${ }^{58,59}$

O trabalho foi dividido em três etapas. Na primeira, foram preparados beads por meio de polimerização em suspensão, empregando CNW e MCM-41 como estabilizantes, visando a obtenção de excipientes para a preparação de comprimidos matriciais de liberação prolongada. Na segunda fase, os beads obtidos foram caracterizados do ponto de vista químico, por meio de análises espectrofotométricas e termogravimétricas. Por último, foram realizados testes físicos, de caráter farmacêutico, que permitem avaliar a aplicabilidade do material como excipiente para compressão direta, uma vez que deve apresentar fluxo livre e boa compressibilidade, parâmetros que podem ser controlados, dentre outros fatores, pelo tamanho e esfericidade dos beads.

\section{PARTE EXPERIMENTAL}

\section{Materiais}

Os monômeros EA, MMA e o BMA, bem como o poli(ácido acrílico) (PAA), procederam da empresa Aldrich, USA. O peróxido de benzoíla (PB) e o peróxido de hidrogênio foram comprados da Vetec (Brasil). Já o sulfato de sódio foi adquirido da Synth (Brasil). As dispersões de nanofibras de celulose e de sílica mesoporosa foram produzidas pelo Laboratório de Físico-Química da Universidade Federal de Minas Gerais.

\section{Caracterização das nanowhiskers de celulose}

As imagens dos NWC foram obtidas por microscopia eletrônica de transmissão (MET) empregando microscópio eletrônico de transmissão Tecnai G2-Spirit - FEI, com aceleração de 120 kV. Dispersões aquosas de NWC foram depositadas em grades eletrônicas microscópicas de cobre revestidas por Formvar (300 mesh). Para melhorar a resolução microscópica, as amostras foram coradas com solução a $2 \%$ de acetato de uranila.

\section{Preparação dos beads pela técnica de polimerização em suspensão}

Para a realização da síntese foi empregado um balão de 250 $\mathrm{mL}$, tritubulado, equipado com um agitador mecânico digital, um termômetro e um sistema de entrada de nitrogênio, para controle do microambiente de polimerização. Uma manta de aquecimento/ resfriamento foi empregada para controlar a temperatura durante a polimerização. A composição qualitativa e quantitativa das formulações é dada na Tabela 1.

$\mathrm{Na}$ preparação dos beads puros (denominados BP), parte do 
Tabela 1. Composição dos sistemas em suspensão

\begin{tabular}{lccc}
\hline Componentes & BP & BNWC & BMCM \\
\hline Água purificada Milli-Q $^{\circledR}$ & $60,00 \mathrm{~mL}$ & $60,00 \mathrm{~mL}$ & $60,00 \mathrm{~mL}$ \\
Poli(ácido acrílico) & $0,20 \mathrm{~g}$ & $0,20 \mathrm{~g}$ & $0,20 \mathrm{~g}$ \\
Sulfato de sódio & $0,20 \mathrm{~g}$ & $0,20 \mathrm{~g}$ & $0,20 \mathrm{~g}$ \\
BMA & $24,00 \mathrm{~g}$ & $24,00 \mathrm{~g}$ & $24,00 \mathrm{~g}$ \\
MMA & $8,00 \mathrm{~g}$ & $8,00 \mathrm{~g}$ & $8,00 \mathrm{~g}$ \\
EA & $8,00 \mathrm{~g}$ & $8,00 \mathrm{~g}$ & $8,00 \mathrm{~g}$ \\
Peróxido de benzoíla & $0,20 \mathrm{~g}$ & $0,20 \mathrm{~g}$ & $0,20 \mathrm{~g}$ \\
NWCs (dispersão $0,6 \% \mathrm{p} / \mathrm{V})$ & - & $0,04 \mathrm{~g}$ & \\
MCM-41 (dispersão $0,6 \% \mathrm{p} / \mathrm{V})$ & - & - & $0,04 \mathrm{~g}$ \\
\hline
\end{tabular}

$\mathrm{BP}=$ beads puros; $\mathrm{BNWC}=$ beads contendo nanowhiskers de celulose; $\mathrm{BMCM}=$ beads contendo MCM-41.

PAA e o sulfato de sódio foram pesados, dissolvidos em água purificada (Milli- ${ }^{\circledR}$ ) e vertidos no reator. Separadamente, o PB foi pesado, dissolvido na mistura dos monômeros (BMA, MMA e EA) e adicionado ao reator. $\mathrm{O}$ aquecimento foi iniciado até a temperatura de $75{ }^{\circ} \mathrm{C}$, sendo mantida constante. Agitação mecânica intensa foi mantida $(750 \mathrm{rpm})$ durante $75 \mathrm{~min}$, tempo após o qual foi adicionado o restante do PAA previamente solubilizado em $5 \mathrm{~mL}$ de água Milli-Q ${ }^{\circledR}$. A temperatura foi ajustada para 90 ${ }^{\circ} \mathrm{C}$ e a agitação foi continuada por $90 \mathrm{~min}$. Após resfriamento, até temperatura ambiente, foram adicionados $0,14 \mathrm{~mL}$ de peróxido de hidrogênio para o consumo de monômeros residuais livres. Os beads assim obtidos foram lavados, sob vácuo, com água em abundância e secos em dessecador, à temperatura ambiente. Quando utilizadas, as dispersões de NWC e de MCM-41 foram incorporadas ao reator, no início do processo. Foram testadas duas concentrações 1 e $0,1 \%$ $\mathrm{p} / \mathrm{p}$ em relação à massa de monômeros. Beads contendo nanocristais de celulose foram designados BNWC e aqueles contendo MCM-41 foram chamados beads BMCM.

\section{Confirmação do processo de polimerização}

As reações de polimerização foram confirmadas através da espectroscopia na região do infravermelho com transformada de Fourier e acessório de reflectância total atenuada (FTIR-ATR). Para avaliação dos beads, os mesmos foram previamente dissolvidos em tetra-hidrofurano e foram preparados filmes por casting, secos a 40 ${ }^{\circ} \mathrm{C}$, por $24 \mathrm{~h}$. Espectros micro-FTIR-ATR, na região de $650-4000 \mathrm{~cm}^{-1}$ foram obtidos, empregando-se microscópio Centaurus acoplado ao espectrofotômetro, utilizando cristal de germânio. Os espectros foram adquiridos com 64 acumulações, com resolução espectral melhor que $4 \mathrm{~cm}^{-1}$, sob purga de nitrogênio seco.

\section{Análises termogravimétricas}

Para pesquisar os eventos térmicos nos copolímeros, foram realizadas análises termogravimétricas (TG e DTG) em aparelho Shimadzu DTG60, em cadinho de alumínio, sob atmosfera de $\mathrm{N}_{2}$, com fluxo de $100 \mathrm{~mL}$ por min e razão de aquecimento de $10^{\circ} \mathrm{C}$ por min.

\section{Avaliação da morfologia dos beads}

A morfologia dos beads foi avaliada por microscopia eletrônica de varredura (MEV). Para tal, as partículas foram recobertas com ouro e as imagens foram adquiridas em equipamento Jeol, modelo JSM-5410, com um potencial de aceleração de $10 \mathrm{kV}$.

\section{Pesquisa do tamanho e distribuição granulométrica das partículas}

Para determinação do tamanho médio das partículas (TMP) e da distribuição granulométrica, foi empregada a análise por difração de raios laser, em granulômetro Cilas, modelo 1064. Para realização das análises, as amostras foram passadas em tamis, dispersas em água e submetidas ao ultrassom por $60 \mathrm{~s}$. A varredura foi realizada entre 1 e $500 \mu \mathrm{m}$. Foram calculados, pelo software, os valores da frequência da distribuição de tamanho $\left(\mathrm{d}_{10 \%}, \mathrm{~d}_{50 \%}\right.$ e $\left.\mathrm{d}_{90 \%}\right)$. Tais valores referem-se à média dos diâmetros de partículas nos intervalos abaixo de 10, 50 e $90 \%$ na curva de distribuição de tamanho. A partir destes dados, o índice de polidispersão (IP) para o tamanho das partículas foi definido, empregando a Equação 1:60,61

$$
\text { IP }=\frac{d_{90 \%}-d_{10 \%}}{d_{50 \%}}
$$

onde: IP = índice de polidispersão; $\mathrm{d}_{90 \%}=$ frequência de tamanho de $90 \%$ das partículas $(\mu \mathrm{m}) ; d_{50 \%}=$ frequência de tamanho de $50 \%$ das partículas $(\mu \mathrm{m}) ; \mathrm{d}_{10 \%}=$ frequência de tamanho de $10 \%$ das partículas $(\mu \mathrm{m})$.

\section{Medidas indiretas e diretas da capacidade de fluxo dos beads}

\section{Medida indireta da fluidez}

As medidas indiretas do fluxo dos excipientes podem ser mensuradas através do cálculo do índice de compressibilidade (IC\%) e do Fator de Hausner (FH), empregando as Equações 2 e 3, respectivamente. Para tal, a densidade aparente e a densidade compactada do material foram inicialmente determinadas, por meio do volume aparente. . $^{29,62,63}$

Para a densidade aparente, $60 \%$ do volume de uma proveta graduada de $100 \mathrm{~mL}$ foi preenchido. A massa referente ao volume ocupado $(60 \mathrm{~mL})$ foi pesada em balança analítica e a densidade aparente foi definida. Para a densidade compactada, foi utilizado um compactador automático Erweka Volumeter Tapped, modelo SVM. A proveta contendo a amostra pesada anteriormente foi acoplada ao volúmetro e o ciclo de batidas foi iniciado. O volume compactado foi anotado após 10 batidas $\left(\mathrm{V}_{10}\right), 500$ batidas $\left(\mathrm{V}_{500}\right)$ e depois de 1250 batidas $\left(V_{1250}\right)$. Em casos onde a diferença entre $V_{500}$ $\mathrm{e}_{1250}$ foi maior que $2 \%$, outro ciclo de 1250 batidas foi realizado. $\mathrm{O}$ procedimento foi repetido até obtenção de variação inferior a $2 \%$. O volume compactado final foi o da última determinação e foi usado no cálculo da densidade compactada. ${ }^{29,62,63} \mathrm{~A}$ partir dos valores das densidades aparente e compactada, foram calculados o IC\% e o FH, empregando as Equações 2 e 3:

$$
\mathrm{IC} \%=\frac{\mathrm{dc}-\mathrm{da}}{\mathrm{dc}}
$$

onde: $\mathrm{IC}=$ índice de compressibilidade (índice de Carr); $\mathrm{d}_{\mathrm{c}}=$ densidade compactada $(\mathrm{g} / \mathrm{mL}) ; \mathrm{d}_{\mathrm{a}}=$ densidade aparente $(\mathrm{g} / \mathrm{mL})$.

$$
\mathrm{FH}=\frac{\mathrm{dc}}{\mathrm{da}}
$$

onde: $\mathrm{FH}=$ Fator de Hausner; $\mathrm{d}_{\mathrm{c}}=$ densidade compactada $(\mathrm{g} / \mathrm{mL})$; $\mathrm{d}_{\mathrm{a}}=$ densidade aparente $(\mathrm{g} / \mathrm{mL})$.

Medida direta da fluidez

A velocidade de fluxo do material e a cotangente do ângulo $\alpha$ 
foram determinados em aparelho Erweka Granulate Flow Tester, tipo GWF, a partir da medida do tempo de escoamento da amostra. Para realização do ensaio, foram pesados $44 \mathrm{~g}$ de cada material, que foram transferidos para o funil de $12 \mathrm{~mm}$, acoplado ao equipamento, no modo sem vibração. O orifício de escoamento do funil foi aberto e o tempo gasto para a massa escoar foi medido. ${ }^{62-64}$ As medidas foram dadas diretamente pelo aparelho. Os parâmetros necessários à interpretação dos resultados obtidos através da análise da velocidade de escoamento são fornecidos na Tabela 2.

Tabela 2. Características de fluxo dos beads conforme o tempo de escoamento e a cotangente de $\alpha$

\begin{tabular}{ccc}
\hline Característica de fluxo & $\begin{array}{c}\text { Tempo de escoamento } \\
\text { (s) }\end{array}$ & Cotangente de $\alpha$ \\
\hline Ótimo & $\leq 10$ & $\leq 0,1$ \\
Suficiente & $10<\mathrm{t}<30$ & $0,1<\operatorname{cotg} \alpha<0,3$ \\
Ruim & $\geq 30$ & $\geq 0,3$ \\
\hline
\end{tabular}

\section{RESULTADOS E DISCUSSÃO}

\section{Caracterização das nanowhiskers de celulose}

As nanofibras de celulose foram obtidas a partir da hidrólise por ácido sulfúrico da polpa de eucalipto, conforme técnica descrita por Mesquita e colaboradores. ${ }^{57}$ A Figura 1 mostra a imagem por MET das NWC e permitiu determinar o comprimento e o diâmetro dos nanocristais: $145 \pm 25$ e $6 \pm 1,5 \mathrm{~nm}$, respectivamente.

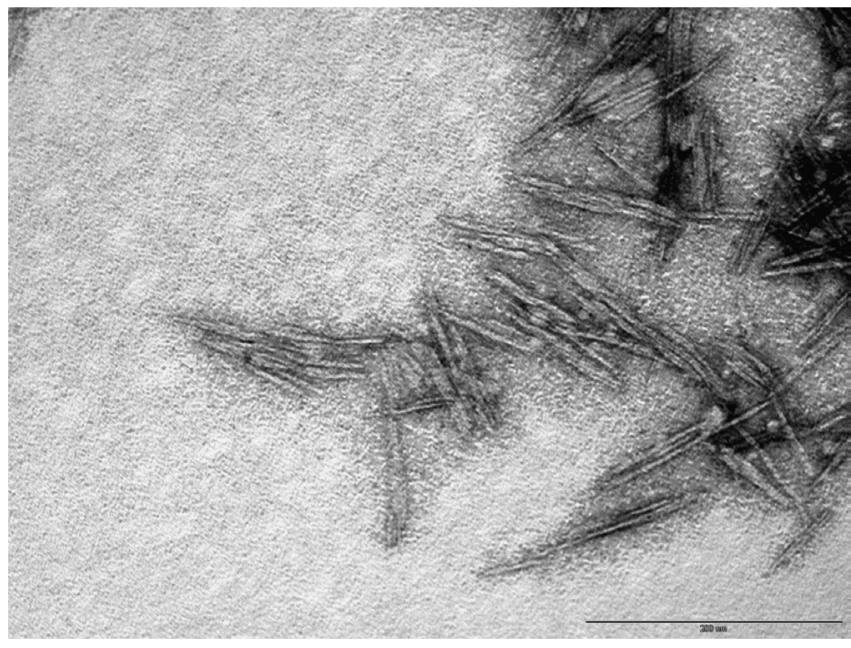

Figura 1. MET das nanowhiskers de celulose. Escala $=200 \mathrm{~nm}$

Espectroscopia no infravermelho por transformada de Fourier (FTIR)

Durante as reações de polimerização, a ligação $\mathrm{C}=\mathrm{C}$ é convertida à ligação C-C, o que pode ser comprovado pela supressão de picos em $1630 \mathrm{~cm}^{-1}$. Os polímeros acrílicos e metacrílicos exibem absorção nas regiões do infravermelho relacionadas à presença de ésteres, sendo característicos dubletos próximos a $2900 \mathrm{~cm}^{-1}$ (estiramentos C-H); banda intensa próxima a $1730 \mathrm{~cm}^{-1}$ (carbonila) e bandas a 1450 e 1385 $\mathrm{cm}^{-1}$ (deformação C-H). ${ }^{65,66}$ Como pode ser visto na Figura 2, houve o desaparecimento das bandas na região de $1630 \mathrm{~cm}^{-1}$, o que indica a ocorrência de polimerização dos monômeros acrílicos durante a formação dos beads. A existência de bandas intensas a 1720 - 1738 $\mathrm{cm}^{-1}$ se refere à vibração de estiramento da carbonila de ésteres conjugados. Dois picos intensos, em 1238 e $1140 \mathrm{~cm}^{-1}$ devem-se ao estiramento C-O, caracterizando grupos ésteres. ${ }^{67}$ Outras bandas bem evidenciadas estão a $1447 \mathrm{~cm}^{-1}$ (vibração simétrica e assimétrica de $\mathrm{CH}_{2}$ e $\mathrm{CH}_{3}$, respectivamente), $1386 \mathrm{~cm}^{-1}$ (vibração simétrica de $\mathrm{CH}_{3}$ ) e $748 \mathrm{~cm}^{-1}$ (rotação de $\mathrm{CH}_{2}$ ). ${ }^{68}$

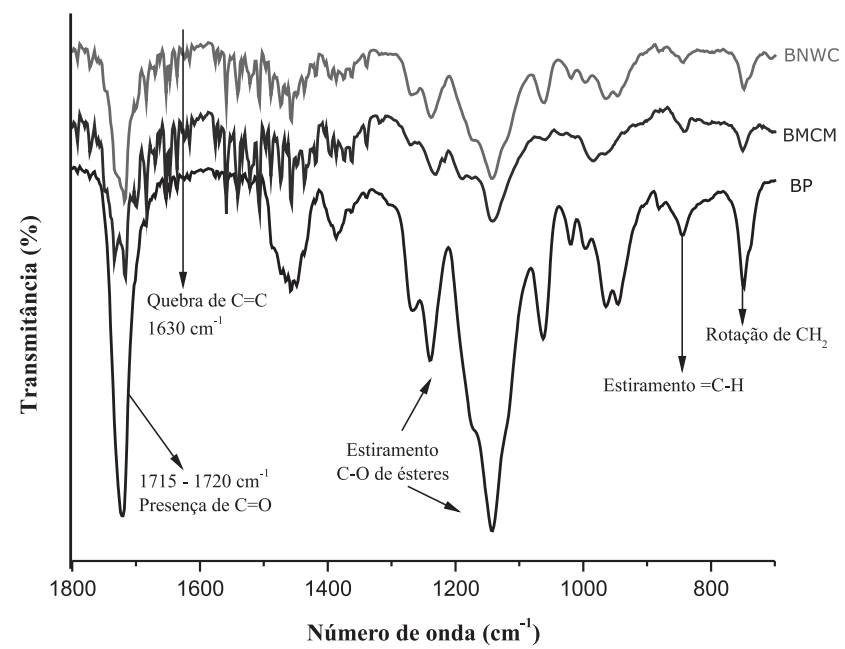

Figura 2. Espectros FTIR dos BP, beads BNWC e beads BMBC (região entre 1800 e $750 \mathrm{~cm}^{-1}$ )

\section{Análise termogravimétrica}

As Figuras 3 e 4 mostram as curvas TG/DTG dos beads puros (a) e dos beads preparados na presença de sílica mesoporosa (b). Ambos apresentaram comportamento semelhante. Os eventos iniciais ocorreram entre temperatura próxima à do ambiente até $160-175^{\circ} \mathrm{C}, \mathrm{com}$ variação de massa de 5\%, correspondente, provavelmente, à perda de água e monômeros livres. A termodecomposição para essas amostras mostra várias etapas em temperaturas entre $100-380^{\circ} \mathrm{C}$, podendo ser relativas à presença de monômeros que permaneceram ocluídos no interior dos beads, que apresentaram granulometria variada. A grande variação da distribuição granulométrica das partículas pode contribuir para tal fato, uma vez que partículas com tamanhos diferentes poderão sofrer termodegradação em tempos diferentes. Cerca de $90 \%$ da massa foi perdida entre $366^{\circ} \mathrm{C}(\mathrm{BMCM})$ e $390{ }^{\circ} \mathrm{C}(\mathrm{BP})$. Partículas menores são mais expostas à degradação térmica devido à sua maior área superficial, gerando assim o comportamento de perda de massa observado para as amostras BMCM e BP.

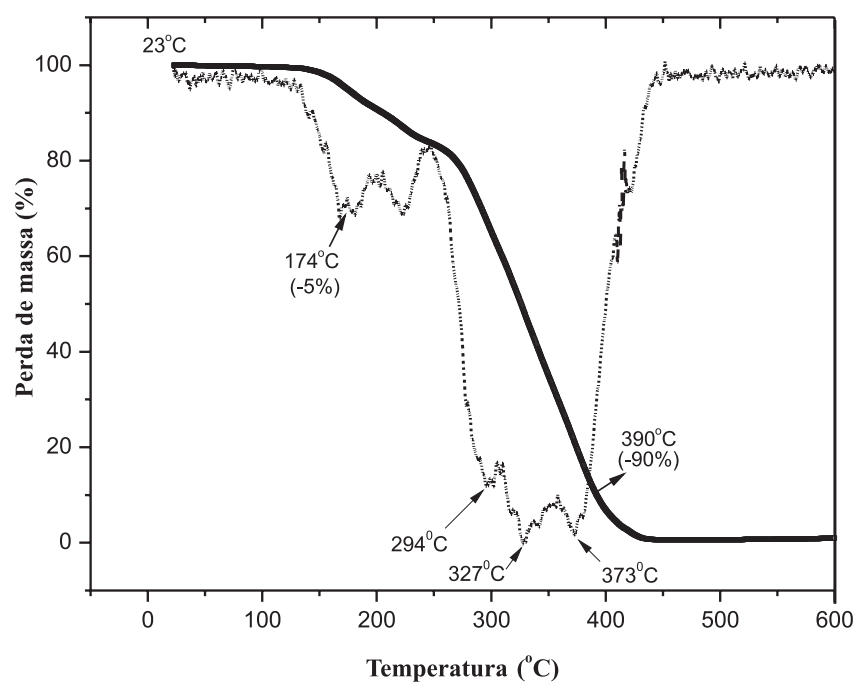

Figura 3. TG e DTG das amostras BP, obtidas sob atmosfera de $N_{2}$ 


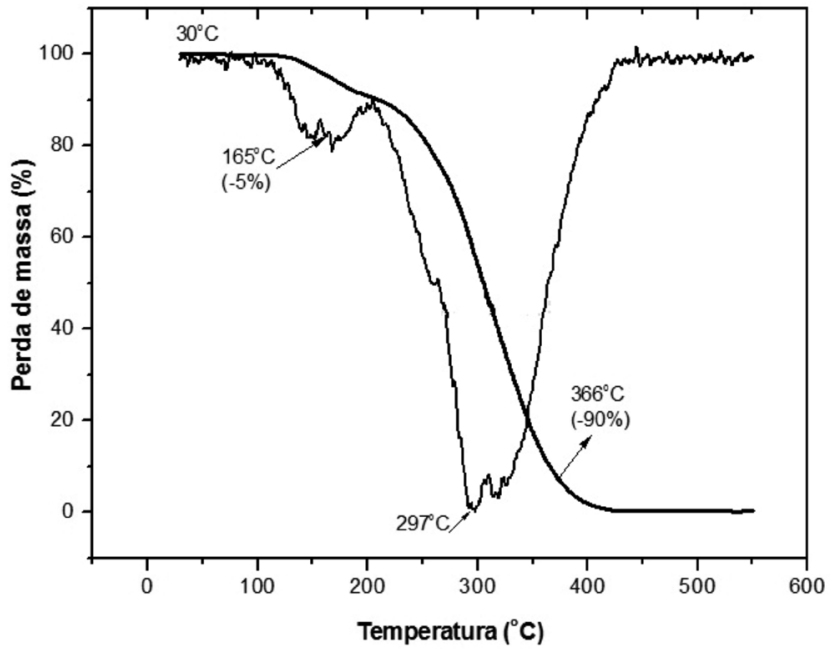

Figura 4. TG e DTG das amostras BMCM, obtidas sob atmosfera de $\mathrm{N}_{2}$

A influência da presença dos NWC nos beads acrílicos se reflete no comportamento térmico dos mesmos. Diferentemente dos sistemas BP e BMCM, a decomposição térmica dos BNWC ocorre em uma só etapa (Figura 5), indicando uma distribuição de tamanho mais estreita dos beads, devido à presença dos NWC usados como coestabilizantes na polimerização em suspensão. Além disso, observa-se uma maior estabilidade térmica dos BNWC em comparação às amostras BMCM, tanto em relação à temperatura de máximo de perda de massa (em torno de $297{ }^{\circ} \mathrm{C}$ para BMCM e de $360{ }^{\circ} \mathrm{C}$ para BNWC), como em relação à temperatura onde se detecta $90 \%$ de perda de massa para as amostras $\left(297{ }^{\circ} \mathrm{C}\right.$ para BMCM e $366^{\circ} \mathrm{C}$ para BNWC). Um relativo aumento de estabilidade térmica também é observado comparando-se as amostras BNWC com BP, visto que a perda de massa para essa última é significativamente maior em temperaturas inferiores a $360{ }^{\circ} \mathrm{C}$. Tais resultados sugerem uma maior estabilidade térmica dos beads BNWC e podem ser explicados pelo fato dos NWCs atuarem de forma efetiva como barreira à difusão de espécies geradas durante a termodegradação, favorecendo o aumento da estabilidade térmica do material.

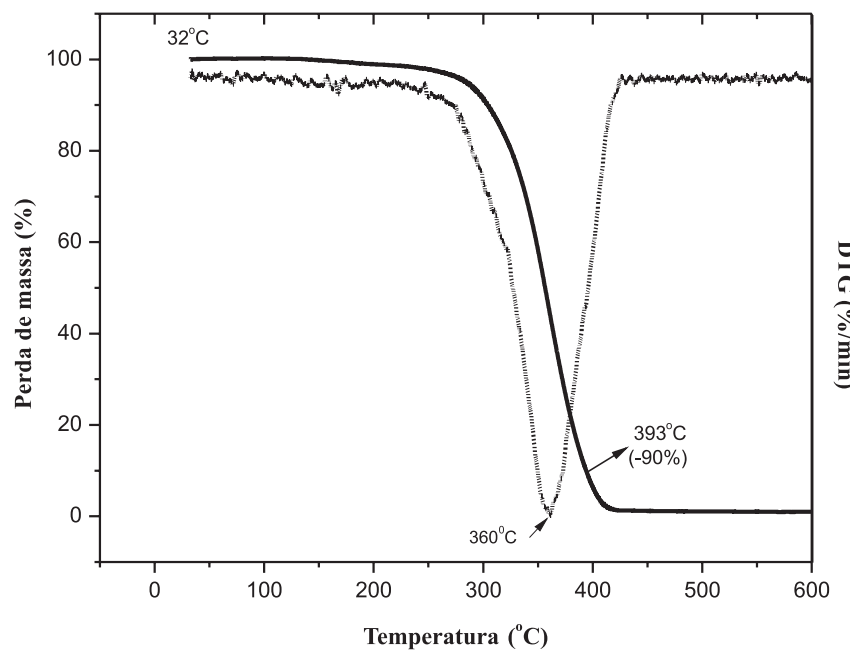

Figura 5. TG e DTG das amostras BNWC, obtidas sob atmosfera de $\mathrm{N}_{2}$

Outra possível explicação para o comportamento térmico diferenciado é que as reações de copolimerização dependem das concentrações relativas dos monômeros, bem como das reatividades relativas de cada um, isto é, da taxa de incorporação de cada um na cadeia.
Se dois monômeros apresentam reatividades diferentes, aquele mais reativo tenderá a se polimerizar mais rápido que o menos reativo, levando à heterogeneidade na composição do copolímero no decorrer da reação. A copolimerização em sistemas ternários é mais complexa que a observada em sistemas binários. ${ }^{69,70}$ Tal fato pode justificar o comportamento dos beads durante o aquecimento: copolímeros com diferentes composições podem ter sido formados e, em consequência, apresentaram degradação térmica em diferentes tempos. Neste caso, os nanocristais de celulose podem ter auxiliado na estabilização do meio reacional, evitando a coalescência e tornando a copolimerização mais homogênea.

\section{Avaliação da morfologia dos beads}

As fotomicrografias obtidas por MEV apresentaram detalhes da morfologia dos beads. Pela análise das imagens pode-se observar que foi possível obter beads esféricos, não porosos, com superfícies lisas e regulares. A formação de aglomerados foi notada nos beads BP, além de grande variação no tamanho das esferas (imagem omitida). Os beads BMCM (Figura 6) apresentaram granulometria elevada e heterogênea. Houve grande diferença entre os tamanhos das partículas preparadas na presença de NWC. A adição de nanofibras de celulose originou esferas de menor tamanho e com menor dispersão granulométrica (Figura 7). Partículas esféricas possuem comportamento superior do ponto de vista do fluxo e compressão, uma vez que tendem a apresentar menos pontos de contato e menor coesividade. Assim, o atrito interparticular e entre as partículas e o equipamento será minimizado, permitindo fácil escoamento e enchimento adequado das matrizes. Tal evidência é de grande importância para o setor farmacêutico. ${ }^{63,64,71-73}$

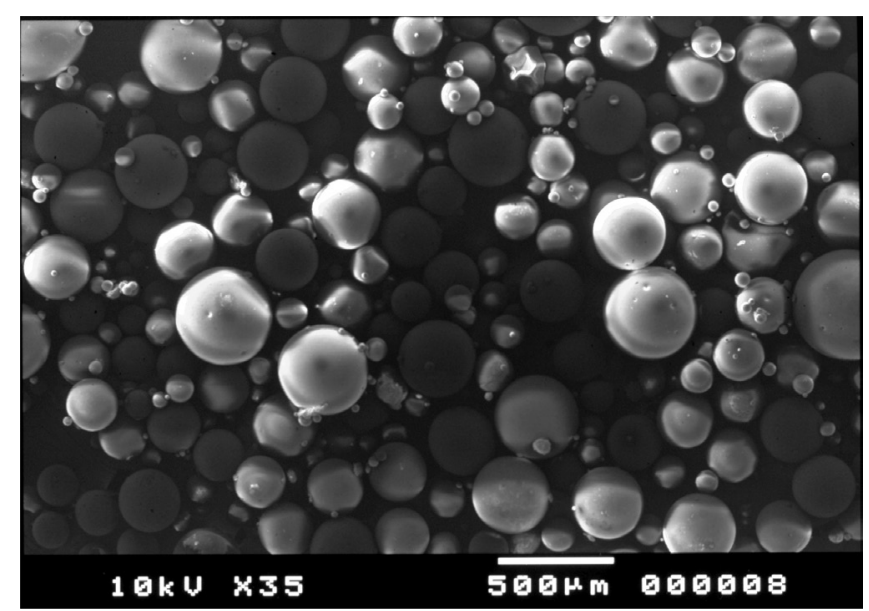

Figura 6. Distribuição granulométrica das amostras BNWC em função da porcentagem acumulada

\section{Determinação do tamanho das partículas e da granulometria}

Sabe-se que pós e/ou grânulos de uso farmacêutico que apresentam grande proporção de pós finos $(<100 \mu \mathrm{m})$, bem como partículas que se apresentam irregulares, tendem a apresentar fluxo reduzido nos equipamentos de mistura e compressão, além de gerar grande quantidade de pó livre durante a compressão. Por outro lado, partículas muito grosseiras causam segregação e preenchimento heterogêneo das matrizes. ${ }^{63,64,71-73}$ Para pesquisar a distribuição granulométrica dos beads foi empregada a técnica de granulometria a laser. Para os beads $\mathrm{BNWC}, 10 \%$ das partículas apresentaram diâmetro menor que $58 \mu \mathrm{m}$ e $50 \%$, tamanho menor que $145 \mu \mathrm{m}$. O diâmetro médio encontrado foi de $200 \mu \mathrm{m}$. O índice de polidispersão calculado foi de 2,50. Para os beads BMCM, 10\% das partículas apresentaram diâmetro 
Tabela 3. Volumes aparentes, em mL, obtidos para os beads BNWC e BMCM

\begin{tabular}{lccccc}
\hline Amostra & Massa $(\mathrm{g})$ & $\mathrm{V}_{0}$ & $\mathrm{~V}_{10}$ & $\mathrm{~V}_{500}$ & $\mathrm{~V}_{1250}$ \\
\hline BNWC & 36,46 & 60 & 57 & 55 & 55 \\
BMCM & 32,94 & 60 & 59 & 58 & - \\
\hline
\end{tabular}

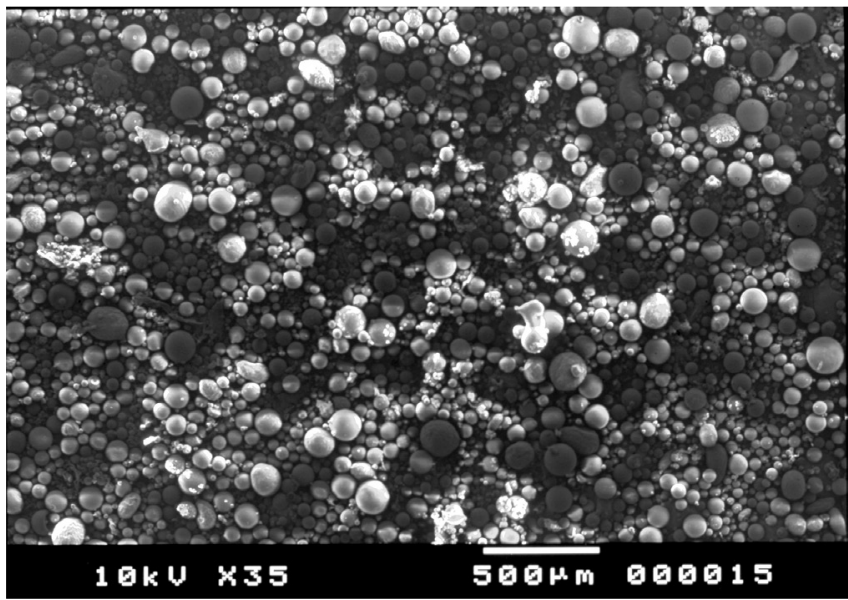

Figura 7. Distribuição granulométrica das amostras BMCM em função da porcentagem acumulada

inferior a $85 \mu \mathrm{m}$ e $50 \%$, tamanho menor que $235 \mu \mathrm{m}$. O diâmetro médio encontrado foi de $298 \mu \mathrm{m}$. O índice de polidispersão calculado foi de 1,80 . Chen e Davis ${ }^{74}$ prepararam microesferas do copolímero poli(hidroxibutirato-hidroxivalerato) (PHBHV) contendo diazepam e consideraram índices de polidispersão próximos a 2 como sendo satisfatórios. Os BPs não foram submetidos aos ensaios de granulometria, uma vez que apresentaram proporção elevada de partículas que ficaram retidas em tamis 42 mesh, inviabilizando a análise.

A classificação dos sólidos particulados de uso farmacêutico, quanto à granulometria, encontra-se descrita nas Farmacopeias. Os sólidos podem ser qualificados de grossos a muito finos, com relação ao diâmetro do tamis no qual ficam retidos, em ensaios de peneiramento. Pós de interesse farmacêutico devem apresentar, preferencialmente, granulometria inferior a $250 \mu \mathrm{m} .{ }^{63,64,71-73}$ Alguns autores consideram que as partículas devem ser inferior a $200 \mu \mathrm{m} .{ }^{75}$ Neste sentido, apesar de apresentar IP mais elevado, os beads BNWC apresentaram melhores resultados, com TMP de $200 \mu \mathrm{m}$, ideal para o uso farmacêutico. Partículas com granulometria mais elevada, como os beads BMCM, podem causar preenchimento irregular das matrizes, originando comprimidos com pesos médios variados e sem uniformidade de conteúdo.

\section{Medidas indiretas e diretas da capacidade de fluxo dos beads}

A partir das análises micromeríticas, parâmetros como o Índice de Carr e o Fator de Hausner podem ser calculados e o comportamento do excipiente frente às condições de manufatura pode ser previsto. Através da interpretação de parâmetros físicos, informações sobre uma das principais características dos sólidos particulados podem ser depreendidas: o comportamento dos mesmos frente ao escoamento. Um bom fluxo do granel influencia, de modo positivo, no processo produtivo, evitando a segregação de misturas e facilitando o enchimento das matrizes na compressora. Em decorrência, exercerá influência no peso médio dos comprimidos, na uniformidade de conteúdo, na dureza e nos resultados dos ensaios de dissolução. $9,63,64,72,76,77$

A densidade aparente $\left(\mathrm{d}_{\mathrm{a}}\right)$ e a densidade compactada $\left(\mathrm{d}_{\mathrm{c}}\right)$ dos sólidos particulados podem ser medidas pela relação entre a massa de dado material e o volume que a mesma ocupa, antes e após compactação e acomodação do material em uma proveta graduada, no equipamento volumeter. Os volumes obtidos nos ensaios de compactação para amostras dos beads BNWC e BMCM encontram-se descritos na Tabela 3. Os beads puros não foram submetidos aos ensaios por apresentarem granulometria muito elevada. A partir de tais resultados foram calculadas o índice de compressibilidade (IC\%) e o Fator de Hausner (FH), apresentados na Tabela 4.

Tabela 4. Medidas do volume aparente, densidade aparente, IC, FH, velocidade de escoamento e ângulo $\alpha$

\begin{tabular}{lcc}
\hline Parâmetros & BNWC & BMCM \\
\hline $\mathrm{d}_{\mathrm{a}}(\mathrm{g} / \mathrm{mL})$ & 0,608 & 0,549 \\
$\mathrm{~d}_{\mathrm{c}}(\mathrm{g} / \mathrm{mL})$ & 0,663 & 0,568 \\
$\mathrm{IC}(\%)$ & 8,30 & 3,34 \\
$\mathrm{FH}$ & 1,091 & 1,035 \\
Velocidade de escoamento $(\mathrm{s})$ & 0,64 & $*$ \\
Cotangente de $\alpha$ & 0,021 & $*$ \\
\hline
\end{tabular}

*Os beads BMCM não escoaram.

O Índice de Carr expressa a capacidade de escoamento e compressão de um sólido. Valores entre 5 e $15 \%$ são indicativos de fluxo excelente e valores entre 12 e $16 \%$, indicam um bom fluxo. Por outro lado, valores maiores que 23 até $35 \%$ são atribuídos a materiais cuja fluidez é pobre. Já o FH, dado pela relação entre a densidade compactada e a aparente, avalia a coesividade do material. Resultados próximos a 1,25 indicam baixa coesividade, enquanto aqueles maiores que 1,5 indicam boa coesividade. Geralmente, se aceita que valores abaixo de 1,25 podem ser correlacionados a um bom fluxo e acima de 1,25 , a um fluxo ruim, uma vez que coesividade e fluxo são inversamente proporcionais. ${ }^{64,78}$ Vale lembrar que, para materiais cujos resultados de IC\% e FH sejam intermediários, a adição de agentes lubrificantes e antiaderentes nas formulações pode melhorar a capacidade de escoamento e a coesividade. ${ }^{63,64,74} \mathrm{Os}$ índices de Carr e de Hausner constituem-se como medidas indiretas do fluxo e não à facilidade ou velocidade com que as partículas escoam. Para avaliar estes dois últimos parâmetros, pode-se determinar o tempo de escoamento e a cotangente do ângulo $\alpha$, consideradas medidas diretas do fluxo. ${ }^{64}$

De acordo com avaliação dos valores do IC\% e do FH pode-se concluir que os beads BNWC apresentaram capacidade de fluxo adequada e baixa coesividade, resultados corroborados pelo pequeno tempo de escoamento $(0,64 \mathrm{~s})$ e pequena cotangente do ângulo $\alpha$ $(0,021)$, sendo adequados ao uso como excipientes em processos de compressão direta. Por outro lado, para os beads BMCM, o IC\% apresentou-se abaixo das especificações e o material não escoou durante o ensaio de medida direta do fluxo, indicando que os beads não são adequados ao processo de compressão. Tais resultados estão de acordo com a determinação da granulometria das partículas: os beads BNWC $(200 \mu \mathrm{m})$ demonstraram TMP adequado ao empacotamento e ao escoamento, ao contrário dos beads BMCM, cujo TMP foi próximo de $300 \mu \mathrm{m}$. A elevada esfericidade do beads BNWC é outro fator que contribui para a confirmação que o excipiente pode ser empregado, com sucesso, em processos de compressão direta. 


\section{CONCLUSÕES}

A polimerização em suspensão mostrou ser eficaz na preparação de copolímeros baseados nos monômeros EA, MMA e BMA, o que foi evidenciado pelos espectros FTIR dos beads, nos quais não foram observados picos a $1630 \mathrm{~cm}^{-1}$, indicando a ocorrência de quebra de ligações $\mathrm{C}=\mathrm{C}$ e a formação de um novo produto. No processo de polimerização em suspensão, quando a reação avança e a viscosidade no sistema se eleva, a tendência à coalescência também aumenta. Evitar a coalescência da fase orgânica, na ausência de tensoativos, é um grande desafio da técnica em suspensão. O uso da concentração de $0,1 \%$ p/p de NWCs (em massa de monômeros) permitiu a estabilidade do meio reacional e a formação de beads BNWC com tamanho de partícula reduzido e elevada esfericidade. Os resultados obtidos nas análises TG/ DTG sugerem uma maior estabilidade térmica dos beads BNWC, o que pode ser explicado pelo fato dos NWC atuarem de forma efetiva como barreira à difusão de espécies geradas durante a termodegradação, favorecendo o aumento da estabilidade térmica do material. Os beads BNWC apresentaram excelentes propriedades de fluxo e compactação, devido, provavelmente, à elevada esfericidade, tamanho médio de partícula de $200 \mu \mathrm{m}$ e densidade compactada apropriada. Portanto, podem ser empregados no desenvolvimento farmacotécnico de comprimidos, em processos de compressão pela via direta. Ao contrário do esperado, a sílica mesoporosa não foi capaz de atuar como agente estabilizante, mesmo contendo, na sua estrutura, um agente surfactante, substância capaz de atuar na redução da tensão superficial e evitar a coalescência. O comportamento da dispersão contendo MCM-41 foi pesquisado, aquecendo-se a dispersão até $70^{\circ} \mathrm{C}$. Durante o aquecimento, verificouse a ocorrência de aglomeração e precipitação da mesma, fato não ocorrido com a dispersão de nanofibras de celulose.

\section{AGRADECIMENTOS}

Ao CNPq e à FAPEMIG pelo apoio financeiro.

\section{REFERÊNCIAS}

1. Villanova, J. C. O.; Sá, V. R.; Excipientes: guia prático para padronização, $2^{\mathrm{a}}$ ed., Pharmabooks: São Paulo, 2009.

2. Pifferi, G.; Restani, P.; Il Farmaco 2003, 58, 541.

3. Gohel, M. C.; Jogani, P. D.; J. Pharm. Pharmaceut. Sci. 2005, 8, 76.

4. Hamman, J. H.; Tarirai, C.; Chim. Oggi 2006, 24, 57.

5. Amidon, G. E.; Garnet, E. P.; Block, L. H.; Moreton, R. C.; Katdare, A.; Lafaver, R.; Sheehan, C.; Pharmacopeial Forum 2007, 33, 1311.

6. Gonnissen, Y.; Remon, J. P.; Vervaet, C.; Eur. J. Pharm. Biopharm. 2007, 67, 220.

7. Beneke, C. E.; Viljoen, A. M.; Hamman, J. H.; Molecules 2009, 14, 2602.

8. Akhgari, A.; Farahmand, F.; Garekani, H. A.; Sadeghi, F.; Vandamme, T. F.; Eur. J. Pharm. Sci. 2006, 28, 307.

9. Bhimte, N. A.; Tayade, P. T.; AAPS PharmSciTech. 2007, 8, E1.

10. Jain, A.; Gupta, Y.; Jain, S. K.; J. Pharm. Pharm. Sci. 2007, 10, 86.

11. Chen, M.; Adv. Drug Deliv. Rev. 2008, 60, 768.

12. Siepmann, F.; Siepmann, J.; J. Controlled Release 2008, 125, 1.

13. Souto-Maior, J. P. A.; Reis, A. V.; Muniz, E. C.; Cavalcanti, O. A.; Int. J. Pharm. 2008, 355, 184.

14. Kim, S.; Kima, J.; Jeon, O.; Kwon, I. C.; Park, K.; Eur. J. Pharm. Biopharm. 2009, 71, 420.

15. Tadros, T.; Adv. Colloid Interface Sci. 2009, 147-148, 281.

16. Builders, P. F.; Bonaventure, A. M.; Tiwalade, A.; Okpako, L. C.; Attama, A. A.; Int. J. Pharm. 2010, 388, 159.

17. Villanova, J. C. O.; Ayres, E.; Carvalho, S. M.; Patrício, P. O.; Vargas, F. P.; Oréfice, R. L.; Eur. J. Pharm. Sci. 2011, 42, 406.
18. Ke, W.; Hsu, T.; Ho, H.; Sheu, M.; Asian J. Pharm. Sci. 2006, 1, 35.

19. Dittgen, M.; Durrani, M.; Lehmann, K.; S.T.P. Pharma Sci. 1997, 6, 403.

20. Wu, C.; McGinity, J. W.; Pharm. Dev. Technol. 2003, 8, 103.

21. Zheng, W.; McGinity, J. W.; Drug Dev. Ind. Pharm. 2003, 29, 357.

22. Dashevsky, A.; Kolter, K.; Bodmeier, R.; Int. J. Pharm. 2004, 279, 19.

23. Villanova, J. C. O.; Dissertação de Mestrado, Universidade de São Paulo, Brasil, 2001.

24. Ojoe, E.; Myauchi, M. E.; Kaneko, T. M.; Velasco, M. V. R.; Consiglieri, V. O.; Braz. J. Pharm. Sci. 2005, 43, 71.

25. Cao, X.; Gao, Q.; Gao, P.; Ding, P.; Gao, Z.; Sun, X.; Asian J. Pharm. Sci. 2007, 2, 29.

26. Khan, M. A.; Reddy, I. K.; S.T.P. Pharm. Sci. 1997, 7, 483.

27. Uchegbu, I.; Schatzlein, A.; Polymers in drug delivery, CRC Press: Boca Raton, 2006.

28. United States Pharmacopeia, $32^{\text {th }}$ ed., United States Pharmacopeial Convention: Rockville, 2009.

29. British Pharmacopoeia, $9^{\text {th }}$ ed., Her Majesty's Stationery Office: London, 2009.

30. Yuan, H. G.; Kalfas, G.; Ray, W. H.; J. Macromol. Sci., Part A: Pure Appl. Chem. 1991, 31, 215.

31. Downding, P. J.; Vincent, B.; Colloids Surf, A 2000, 161, 259.

32. Santos, J. C.; Lopes, C. N.; Reis, M. M.; Giudici, R.; Sayer, C.; Machado, R. A. F.; Araújo, P. H. H.; Braz. J. Chem. Eng. 2008, 25, 399.

33. Hashim, S.; Brooks, B. W.; Chem. Eng. Sci. 2002, 57, 3703.

34. Hashim, S.; Brooks, B. W.; Chem. Eng. Sci. 2004, 59, 2321.

35. Maggioris, D.; Goulas, A.; Alexopoulos, A. H.; Chatzi. E. G.; Kiparissides, C.; Chem. Eng. Sci. 2000, 55, 4611.

36. Kichatov, B. V.; Korshunov, A. M.; Assorova, P. V.; Theor. Found. Chem. Eng. 2003, 37, 306.

37. Jahanzad, F.; Sajjadi, S.; Brooks, B. W.; Ind. Eng. Chem. Res. 2005, 44, 4112.

38. Kotoulas, C.; Kiparissides, C.; Chem. Eng. Sci. 2006, 61, 332.

39. Alexopoulos, A. H.; Kiparissides, C.; Chem. Eng. Sci. 2007, 62, 3970.

40. Jahanzad, F.; Sajjadi, S.; Yianneskis, M.; Brooks, B. W.; Chem. Eng. Sci. 2008, 63, 4412 .

41. He, Y.; Howes, T.; Litster, J.; Ko, G. H.; Physicochem. Eng. Aspects 2002, 207, 89.

42. Georgiadou, S.; Brooks, B. W.; Chem. Eng. Sci. 2006, 61, 6892.

43. Guerero-Sanchez, C.; Erdmenger, T.; Sereda, P.; Wouters, D.; Schubert, U. S.; Chem. Eur. J. 2006, 12, 9036.

44. Wolters, D.; Meyer-Zaika, M.; Bandermann, F.; Macromol. Mater. Eng. 2001, 286, 94.

45. Aveyard, R.; Clin,t J. H.; Horozov, T. S.; Phys. Chem. Chem. Phys. 2003, 5, 2398 .

46. Sacanna, S.; Kegel, W. K.; Philipse, A. P.; Phys. Rev. Lett. 2007, 98, 1.

47. Tarimala, S.; Ranabothu, S. R.; Vernetti, J. P.; Dai, L. L.; Langmuir 2004, 20, 5171.

48. He, X. D.; Ge, X. W.; Liu, H. R.; Zhou, H. X.; Zhang, Z. C.; Colloids Surf., A 2007, 301, 80.

49. Gao, Q.; Wang, C.; Liu, H.; Wang, C.; Liu, X.; Tong, Z.; Polymer 2009, 50, 2587.

50. Gao, Q.; Wang, C.; Liu, H.; Chen, Y.; Tong, Z.; Polym. Chem. 2010, 1, 75.

51. Voor, D. J.; Ming, W.; van Herk, A.; Macromolecules 2006, 39, 2137.

52. Morishita, C.; Kawaguchi, M.; Colloids Surf., A 2009, 335, 138.

53. Wang, C.; Zhang, C.; Li, Y.; Chen, Y.; Tong, Z.; React. Funct. Polym. 2009, 69, 750 .

54. Bachinger, A.; Kickelbick, G.; Monatsh. Chem. 2010, 141, 685.

55. Ma, H.; Luo, M.; Sanyal, S.; Rege, K.; Dai, L. L.; Materials 2010, 3, 1186.

56. Silva, R.; Haraguchi, S. K.; Muniz, E. C.; Rubira, A. F.; Quim. Nova 2009, 32, 661 .

57. Mesquita, J. P.; Donnici, C. L.; Pereira, F. V.; Biomacromolecules 2010, $11,473$. 
58. Schmidt-Winkel, P.; Lukens, W. W.; Yang, P.; Margolese, D. I.; Lettow, J. S.; Ying, J. Y.; Stucky, G. D.; Chem. Mater. 2000, 12, 686.

59. Sayari, A.; Hamoudi, S.; Chem. Mater. 2001, 13, 3151.

60. Torrado, J. J.; Illum, L.; Davis, S. S.; Int. J. Pharm. 1989, $51,85$.

61. Khunawattanakul, W.; Puttipipatkhachorn, S.; Rades, T.; Pongjanyakul, T.; Int. J. Pharm. 2008, 161, 149.

62. Schüssele, A.; Bauer-Brandl, A.; Int. J. Pharm. 2003, 257, 301.

63. Swarbrick, J.; Encyclopedia of Pharmaceutical Technology, $3^{\text {rd }}$ ed., Informa Healthcare: New York, 2007.

64. Aulton, M.; Aulton's Pharmaceutics: the design and manufacture of medicines, $3^{\text {rd }}$ ed., Elsevier: Canada, 2007.

65. Sapozhnikov, D. A.; Volkova, T. V.; Sakharova, A. A.; Gasanov, R. G.; Voytekunas, V. Y.; Abadie, M. J. M.; Sanchez, J.; Vygodskii, Y. S.; Polym. Sci. Ser. B 2008, 51, 1.

66. Sapozhnikov, D. A.; Volkova, T. V.; Sakharova, A. A.; Gasanov, R. G.; Voytekunas, V. Y.; Abadie, M. J. M.; Sanchez, J.; Vygodskii, Y. S.; Int J. Pol. Sci. (2009), doi:10.1155/2009/527046.

67. Patel, M. V.; Dolia, M. B.; Patel, J. N.; Patel, R. M.; React. Funct. Polym. 2005, 65, 195.
68. Silverstein, R. M.; Webster, F. X.; Kiemle, D.; Spectrometric identification of organic compounds, $7^{\text {th }}$ ed., John Wiley \& Sons: New York, 2007.

69. Anderson, C. D.; Daniels, E. S.; Rapra Rev. Rep. 2003, 14, 3.

70. Riddle, E. H.; Monomeric acrylic esters, Reinhold Publishing Co: New York, 1954.

71. Brittain, H. G.; Physical characterization of pharmaceutical solids, Marcel Dekker: New York, 1995.

72. Allen, L. V.; Popovich, N. G.; Ansel, H. C.; Ansel's pharmaceutical dosage forms, $8^{\text {th }}$ ed., Lippincot Williams \& Wilkins: Baltimore, 2008.

73. Sinko, P. J.; Martin's physical Pharmacy and Pharmaceutical sciences, $5^{\text {th }}$ ed., Lippincott Williams \& Wilkins: Baltimore, 2006.

74. Chen, J.; Davis, S. S.; J. Microencapsulation 2002, 19, 191.

75. Shekunov, B. Y.; Chattopadhyay, P.; Tong, H. H. Y.; Chow, A. H. L.; Pharm. Res. 2007, 24, 203.

76. Emeje, M. O.; Kunle, O.; Ofoefule, S. I.; Acta Pharm. 2006, 56, 325.

77. Battacharyya, S. B.; Battacharyya, I.; Patro, N.; Asian J. Pharm. 2010, $4,24$.

78. Kwakye, K.; Asantewaa, Y.; Kipo, S. L.; Int. J. Pharm. Pharm. Sci. 2010, 2, 105. 